\title{
Efecto del extracto hidroalcohólico liofilizado de hojas de Bixa orellana (achiote), en la secreción gástrica de ratas
}

\author{
Oscar Huamán ${ }^{1}$, Inés Arnao ${ }^{1}$, Elsa Béjar ${ }^{1}$, Miguel Sandoval ${ }^{1}$
}

Resumen

Palabras clave

\begin{abstract}
Objetivo: Determinar el efecto del extracto hidroalcohólico de hojas Bixa orellana (achiote) sobre los niveles de grupos sulfidrilos no proteicos (GS-NP), moco gástrico, pH y acidez total de la secreción gástrica. Diseño: Estudio prospectivo, analíticoexperimental y transversal. Lugar: Laboratorios del Centro de Investigación de Bioquímica y Nutrición de la Facultad de Medicina de la Universidad Nacional Mayor de San Marcos. Material biológico: Para evaluar el efecto del extracto sobre la mucosa gástrica se utilizó el modelo de la ligadura pilórica, propuesto por Vissher y modificado por Sandoval. En la determinación de GS-NP se utilizó el método de Sadlak y Lindsay; la producción de moco gástrico fue según Corne, el pH por potenciómetro y la acidez total por titulación con $\mathrm{NaOH}$. Principales medidas de resultados: Niveles de grupos sulfidrilos no proteicos (GS-NP), moco gástrico, pH y acidez total. Resultados: El extracto hidroalcohólico de hojas de Bixa orellana incrementó los GS-NP en 133 y 168\% (p<0,01), a las dosis de 100 y $200 \mathrm{mg} / \mathrm{kg}$, y la producción de moco, en 39,4\% y 44,9\%, respectivamente, a las dosis de 200 y 400 $\mathrm{mg} / \mathrm{kg}(\mathrm{p}<0,01)$. Sin embargo, solo en el grupo que recibió la dosis de $400 \mathrm{mg} / \mathrm{kg}$ de peso se produjo un descenso de $\mathrm{pH}$ de forma significativa $(p<0,05)$. Por otro lado, la acidez total a la dosis de extracto 100, 200 y $400 \mathrm{mg} / \mathrm{kg}$ presentaron un incremento de 62,3\%, 58,2\% y 166,6\%, respectivamente ( $<<0,01)$. Conclusiones: El tratamiento con extracto hidroalcohólico de Bixa orellana incrementa la producción de GS-NP y moco gástrico, sin inhibición de la acidez total.

Bixa orellana; mucosa gástrica; úlcera gástrica; extractos vegetales.
\end{abstract}

Bixa orellana (achiote) hydroalcoholic liofilized leaves effect on rats' gastric secretion

Abstract

Objective: To determine the effect of Bixa orellana (achiote) leaves hydroalcoholic extract on non-protein sulphydryles groups (NP-SG), gastric mucus $p H$ and gastric secretion total acidity. Design: Prospective, analytical-experimental and transversal study. Setting: Biochemistry and Nutrition Research Centre, Faculty of Medicine, Universidad Nacional Mayor de San Marcos. Biologic material: To determine the effect of the extract on gastric mucosa we used the pyloric ligation model proposed by Vissher and modified by Sandoval. For NP$S G$ determination Sadlak and Lindsay method was used, Corne's method for gastric mucus production, $\mathrm{pH}$ and total acidity potentiometer by titration with $\mathrm{NaOH}$. Main outcome measures: Levels of sulphydryle non-protein

1 Centro de Investigación de Bioquímica y Nutrición, Facultad de Medicina, Universidad Nacional Mayor de San Marcos. Lima, Perú. groups (NP-SG), gastric mucus, $p H$ and total acidity. Results: Bixa orellana leaves hydroalcoholic extract $N P-S G$ increased in $133 \%$ and $168 \%(p<0,01)$ at 100 and $200 \mathrm{mg} / \mathrm{kg}$ doses and increased mucus production in $39,4 \%$ and $44,9 \%$ respectively at 200 and $400 \mathrm{mg} / \mathrm{kg}$ doses $(p<0,01)$. Only in the group receiving the $400 \mathrm{mg} /$ $\mathrm{kg}$ dose there was a significant $\mathrm{pH}$ decrease $(p<0,05)$. On the other hand, at 100, 200 and $400 \mathrm{mg} / \mathrm{kg}$ extract doses total acidity increased 62,3\%, 58,2\% and 166,6\% respectively $(p<0,01)$. Conclusions: Treatment with Bixa orellana leaves extract increases the production of mucus and GS-NP but does not inhibit total acidity.

Key words: Bixa orellana; gastric mucosa; stomach ulcer; plant extracts.

\section{INTRODUCCIÓN}

La úlcera gástrica es la pérdida de sustancia de la pared gástrica debido a la rotura en el epitelio entre los mecanismos defensivos de la mucosa y la agresión del jugo gástrico, que en condiciones 
normales no ocurre. Se asocia a múltiples factores. Entre ellos mencionaremos la hipersecreción del ácido, anomalía pilórica, aumento en la secreción de gastrina, trastorno de la motilidad del píloro, disminución del moco protector de la mucosa gástrica, el uso inadecuado de antiinflamatorios no esteroideos (AINES), presencia de Helicobacter pylori, estrés y estilo de vida $\left({ }^{1}\right)$, siendo este último uno de los factores más frecuentes en los pacientes que presentan esta enfermedad.

En la actualidad, la terapéutica de esta enfermedad incluye diversos tratamientos, entre ellos, inhibidores de receptores $\mathrm{H}_{2}$, antiácidos, antibacterianos, bloqueadores de la bomba de $\mathrm{H}^{+} / \mathrm{K}^{+} \mathrm{ATPa}-$ sa, anticolinérgicos y últimamente los compuestos naturales, como flavonoides $\left({ }^{2}\right)$.

Desde tiempos milenarios, el empleo de plantas con finalidad terapéutica ha sido una práctica que el hombre continúa hasta hoy. Sin embargo, el avance de la tecnología, los cambios culturales externos y, en el caso propio del Perú, la falta de un lenguaje gráfico, determinó que gran parte de estos conocimientos, productos de las culturas preinca e inca se perdieran de forma irreversible.

En nuestro país, el uso de plantas medicinales con atribuciones antiulcerosas es muy difundido y por ello se están llevando a cabo diferentes estudios sobre su acción farmacológica. Como ejemplo tenemos los estudios realizados con Buddleia globosa (matico) $\left(^{3}\right)$, Piper angustifolium (matico) $\left({ }^{4,5}\right)$, Crotón palanostigma (sangre de grado) y Aloe vera (sábila) ${ }^{6}$ ).

La Bixa Orellana (achiote) es otra de las plantas a las que se le atribuye efectos antiulcerosos, pero su uso se encuentra limitado a la zona de la selva alta, siendo el efecto antiinflamatorio y 'refrescante del estómago' los más conocidos en dicha región.

El uso terapéutico que se atribuye a la planta es muy variado y depende de la parte que se va a utilizar. La información recogida por entrevista, durante la recolección de la muestra y por diversas publicaciones, menciona que se emplea las semillas para tratar bronquitis, hemorroides, para las quemaduras, antidiarreico, antiemético, antidia- bético y expectorante $\left(^{7}\right)$. La raíz es considerada antidisentérica, antivenérea e hipoglicemiante y las hojas como desinflamatorio bucal, diurético, antiinflamatorio prostático (que es una de las propiedades más difundida), enfermedades del hígado $\left({ }^{8}\right)$. En la medicina popular venezolana se emplea las hojas en decocción para curar las enfermedades del hígado y 'limpiar la sangre'. Cuando se las aplica en la frente y sienes actúan como refrescante, en el 'dolor de muela' y de cabeza $\left(^{8}\right)$.

Nuestro objetivo en el presente estudio fue evaluar el efecto del extracto hidroalcohólico de las hojas de Bixa orellana sobre la mucosa gástrica, determinando la producción de grupos sulfidrilos no proteicos (GS-NP), moco gástrico y sobre la secreción gástrica, midiendo el $\mathrm{pH}$ y la acidez total.

\section{MÉTODOS}

Se realizó un estudio prospectivo, analíticoexperimental y transversal en los laboratorios del Centro de Investigación de Bioquímica y Nutrición de la Facultad de Medicina de la Universidad Nacional Mayor de San Marcos.

Para el presente trabajo se usó las hojas de Bixa orellana (achiote) recolectadas en la Región Amazonas, distrito de La Peca, que fueron molidas y maceradas en una solución de etanol al $80 \%$ en una proporción de 1:10, en botella de color ámbar con tapa, y con agitación manual por 15 minutos al día, por un tiempo de 15 días. Luego, se filtró, se eliminó el etanol en la estufa a $40^{\circ} \mathrm{C}$, previo a su liofilización, y se conservó en un desecador a $-4^{\circ} \mathrm{C}$.

Para evaluar el efecto in vivo del extracto, se utilizó 45 ratas machos Holtzman de un peso de $190 \mathrm{~g} \pm 17,1$, con un tiempo de adaptación de 7 días, recibiendo alimentación balanceada, agua $a d$ libitum, a una temperatura de 20 a $22^{\circ} \mathrm{C}$ y condiciones ambientales controladas.

Las ratas fueron mantenidas en ayuno de 24 horas, antes del ensayo con dextrosa $5 \%$ ad libitum, y fueron distribuidas aleatoriamente en 5 grupos de 9 ratas, a las que se les administró por vía oro- 
gástrica lo siguiente: grupo I, suero fisiológico; grupo II, ranitidina $100 \mathrm{mg} / \mathrm{kg}$; grupo III, extracto hidroalcohólico de Bixa orellana $100 \mathrm{mg} / \mathrm{kg}$ : grupo IV, extracto hidroalcohólico de Bixa orellana $200 \mathrm{mg} / \mathrm{kg}$; y, grupo V, extracto hidroalcohólico de Bixa orellana $400 \mathrm{mg} / \mathrm{kg}$. Luego de una hora, se anestesió a las ratas con vapores de éter y se procedió a realizar la ligadura pilórica, siguiendo el modelo propuesto por Vissher, modificado por Sandoval $\left({ }^{9}\right)$.

Para la determinación de grupos sulfidrilos no proteicos (GS-NP) y moco, se utilizó dos secciones de la parte glandular del estómago, y para la medición del pH y acidez total se recolectó el contenido gástrico, que fue mantenido en refrigeración a $4^{\circ} \mathrm{C}$.

Se determinó los GS-NP por el método de Sedlak y Lindsay $\left({ }^{2}\right)$, teniendo como principio el reconocimiento de grupos sulfidrilos por el ácido 5,5'-dithiobis (2-nitrobenzoico), el cual fue leído a $412 \mathrm{~nm}$, en un espectrofotómetro. Para determinar la concentración de GS-NP, se preparó una curva de calibración usando glutatión (GSH) como estándar, en el rango de 3,0 a 50,0 $\mu \mathrm{g} / \mathrm{mL}\left({ }^{2}\right)$. Se expresó los resultados en $\mu \mathrm{g}$ de GS-NP/mL/g de tejido y en porcentaje de incremento de GS-NP, mediante la siguiente fórmula:

\section{$\%$ de incremento de GS-NP = (IGSt-IGSc)/IGSc $\times 100 \%$}

Siendo IGSt: índice de grupo sulfidrilos no proteico del tratamiento, y IGSc: índice de grupo sulfidrilos no proteico del control.

La producción de moco gástrico se determinó mediante el método espectrofotométrico modificado de Corne $\left({ }^{2}\right)$, teniendo como principio la adsorción del Alciam blue por el moco gástrico, que luego fue extraído con una solución de $\mathrm{MgCl}_{2}$, para posteriormente ser leído a $598 \mathrm{~nm}$. Se preparó una curva de calibración de Alcian blue, con concentraciones de 3,0 a 50,0 $\mu \mathrm{g} / \mathrm{mL}$. Los resultados fueron expresados en $\mu \mathrm{g}$ Alcian blue $/ \mathrm{mL} / \mathrm{g}$ de tejido y en porcentaje de incremento de moco gástrico, según la siguiente fórmula:

\section{$\%$ de incremento de mucus $=($ IMGt-IMGc) $) / I M G c \times 100 \%$}

Siendo IMGt: índice de moco gástrico del tratamiento, e IMGc: índice de moco gástrico del control.
Para evaluar el contenido gástrico, se usó el sobrenadante obtenido tras la centrifugación a $600 \mathrm{~g}$ por 10 minutos. La determinación de $\mathrm{pH}$ se realizó utilizando un potenciómetro con una precisión de $+/-0,01 \mathrm{pH}$. La determinación de la acidez total se determinó mediante titulación con $\mathrm{NaOH} 0,05$ $\mathrm{N}$. Los resultados fueron expresados en $\mathrm{mEq}-\mathrm{H}^{+} / \mathrm{L}$ y porcentaje de incremento de acidez (IAT):

$\%$ de incremento de la acidez total=(IATt-IATC)/IATc $\times 100 \%$

Siendo IATt: índice de acidez total del tratamiento, e IATc: índice de acidez total del control.

Todos los resultados analizados fueron expresados en promedio y desviación estándar; los datos fueron sometidos a análisis de varianza (ANVA). Para el estudio, se consideró valores de significancia con un $p<0,01 \mathrm{y} / \mathrm{o} p<0,05$.

\section{RESULTADOS}

El efecto del extracto hidroalcohólico de Bixa orellana sobre la mucosa gástrica se observó en las diferentes dosis administradas, tanto en la producción de GS-NP como de moco gástrico.

Como se puede observar en la Tabla 1, los grupos III y IV presentaron un incremento de GS-NP en $133 \%$ y $168 \%$, con respecto al control $(p<0,01)$. Estos resultados también fueron comparados con los obtenidos con la ranitidina, existiendo una diferencia significativa $(p<0,01)$. Sin embargo, a dosis de extracto $400 \mathrm{mg} / \mathrm{kg}$ solo hubo un incremento en $25,2 \%$, con respecto al control, dato que no resultó estadísticamente significativo.

Con respecto a la producción de moco se observó un incremento en los grupo IV y V $(39,4$ y $44,9 \%$, respectivamente), comparado con el control, existiendo diferencia significativa $(p<0,01)$, tal como se aprecia en la Tabla 1 . Al realizar el análisis de los datos se observa que no existe diferencia estadística entre el grupo control y el grupo que recibió el tratamiento con ranitidina $(6,8 \%)$.

Al comparar el grupo que recibió la ranitidina con los grupos IV y V, se observa una mayor 
Tabla 1. Determinación de GS-NP y moco inducidos por extracto de hojas.

\begin{tabular}{|c|c|c|c|c|}
\hline \multirow{2}{*}{$\begin{array}{c}\text { Grupo } \\
\text { (9 ratas c/u) }\end{array}$} & \multicolumn{4}{|c|}{ Mucosa gástrica } \\
\hline & $\begin{array}{c}\text { GS-NP } \\
\mu \mathrm{g} \mathrm{GSH} / \mathrm{mL} / \mathrm{g}\end{array}$ & $\begin{array}{l}\text { Incremento } \\
\text { GS-NP (\%) }\end{array}$ & $\begin{array}{c}\text { Moco gástrico } \\
\mu \mathrm{g} \mathrm{AB} / \mathrm{mL} / \mathrm{g}\end{array}$ & $\begin{array}{l}\text { Incremento } \\
\text { de moco (\%) }\end{array}$ \\
\hline Grupo I (Control ) & $16,2 \pm 3,8$ & - & $27,5 \pm 6,2$ & - \\
\hline Grupo II (Ranitidina 100 mg/kg) & $17,3 \pm 2,7$ & 6,6 & $29,4 \pm 6,7$ & 6,8 \\
\hline Grupo III (Extracto 100 mg/kg) & $37,72 \pm 10,29$ a,b & 133,1 & $21,1 \pm 4,9$ & $-23,2$ \\
\hline Grupo IV (Extracto 200 mg/kg) & $43,4 \pm 10,9$ & 168,1 & $38,4 \pm 5,8^{\mathrm{a}, \mathrm{b}}$ & 39,4 \\
\hline Grupo V (Extracto 400 mg/kg) & $20,3 \pm 6,8$ & 25,2 & $39,9 \pm 10,1^{\mathrm{a}, \mathrm{c}}$ & 44,9 \\
\hline
\end{tabular}

(a) $p<0,01$ (comparadas con el control)

(b) $p<0,01$ (comparadas con la ranitidina)

(c) $p<0,05$ (comparadas con la ranitidina)

respuesta al tratamiento con el extracto de Bixa orellana a esas dos dosis $(p<0,01$ y $p<0,05$, respectivamente).

En el tratamiento con las dosis de $100 \mathrm{mg} / \mathrm{kg}$, se observa una disminución de la barrera protectora, que incluso está por debajo del grupo control $(-23,2 \%)$, no existiendo diferencia estadísticamente significativa.

El tratamiento con ranitidina no indujo a una variación significativa del $\mathrm{pH}$ en los grupos tratados con extracto hidroalcohólico de Bixa orellana, a las dosis de 100 y $200 \mathrm{mg} / \mathrm{kg}$. Sin embargo, a dosis de $400 \mathrm{mg} / \mathrm{kg}$ sí redujo el $\mathrm{pH}$, con respecto al control $(p<0,05)$, tal como se aprecia en la Tabla 2.

El tratamiento con ranitidina mantuvo los niveles de acidez total en el contenido gástrico, con respecto al control.
Como se puede apreciar en la Tabla 2, los grupos que recibieron el tratamiento con el extracto a diferentes dosis $(100,200$ y $400 \mathrm{mg} / \mathrm{kg})$ incrementaron la acidez total en el jugo gástrico, en $62,3 \%$, $58,2 \%$ y $166,6 \%$, respectivamente, con respecto al control y la ranitidina $(p<0,01)$.

\section{DISCUSIÓN}

Uno de los mecanismos que se postula como causante de las lesiones en los tejidos producidos en la úlcera gástrica es el incremento de los radicales libres; es por ello nuestro interés en determinar un metabolito capaz de neutralizar dichas sustancias.

El glutatión está presente en altas concentraciones en la mucosa gástrica de ratas, perros y en el hombre. Está indicado como un posible mediador

Tabla 2. Evaluación del pH y acidez total en el jugo gástrico de ratas.

\begin{tabular}{|c|c|c|c|}
\hline \multirow{2}{*}{$\begin{array}{c}\text { Grupo } \\
\text { (9 ratas c/grupo) }\end{array}$} & \multicolumn{3}{|c|}{ Jugo gástrico } \\
\hline & $\begin{array}{c}\text { Acidez libre } \\
\text { pH } \\
\end{array}$ & $\begin{array}{l}\text { Acidez total } \\
{[\text { meq } \mathrm{H}+/ \mathrm{L}]}\end{array}$ & $\begin{array}{c}\text { \% incremento de la } \\
\text { acidez total }\end{array}$ \\
\hline Grupo I (Control ) & $1,5 \pm 0,1$ & $39,5 \pm 7,6$ & - \\
\hline Grupo II (Ranitidina 100 mg/kg) & $1,8 \pm 0,2^{\mathrm{a}}$ & $40,0 \pm 7,6$ & 1,3 \\
\hline Grupo III (Extracto 100 mg/kg) & $1,4 \pm 0,1^{\mathrm{c}}$ & $64,1 \pm 10,5^{\mathrm{a}, \mathrm{c}}$ & 62,3 \\
\hline Grupo IV (Extracto 200 mg/kg) & $1,5 \pm 0,1^{\mathrm{c}}$ & $62,5 \pm 16,9^{\mathrm{a}, \mathrm{c}}$ & 58,2 \\
\hline Grupo V (Extracto 400 mg/kg) & $1,3 \pm 0,1^{\mathrm{b}, \mathrm{c}}$ & $105,3 \pm 7,9^{\mathrm{a}, \mathrm{c}}$ & 166,6 \\
\hline
\end{tabular}

(a) $p<0,01$ (comparadas con el control + )

(b) $p<0,05$ (comparadas con la ranitidina)

(c) $p<0,01$ (comparadas con la ranitidina) 
citoprotector, al inducir prostaglandinas (PG) $\left({ }^{10}\right)$. La concentración del glutatión disminuye cuando el epitelio gástrico es injuriado por agentes químicos y estrés. También, se comunica que el incremento de prostaglandinas estimula el aumento de GS-NP en dicho tejido, siendo el glutatión el mayor componente $\left({ }^{10}\right)$.

En un estudio realizado con una fracción enriquecida de flavonoides de Alchornea castaneafolea, se observó un incremento en los niveles de prostaglandinas, lo que evidencia un probable efecto inductivo de parte de los flavonoides presentes, tales como la quercetina, mirecitina y amenflavona ${ }^{(11)}$. De todos estos metabolitos, la quercetina fue identificada en las hojas de Bixa orellana $\left({ }^{12}\right)$. No es solo la presencia de quercetina, sino también de taninos y carotenoides -como la bixina y la norbixina presentes en Bixa orellana- que justificarían su actividad citoprotectora, al inducir la producción de prostaglandinas y GS-NP.

En el presente estudio encontramos un incremento de los GS-NP estadísticamente significativo, a las dosis de 100 y $200 \mathrm{mg} / \mathrm{kg}$. Con ésta última se obtiene la máxima producción, lo que ayudaría en la protección contra las especies reactivas de oxígeno (ROS) que se producen como consecuencia del proceso inflamatorio, agudizándose por la infiltración mononuclear en las diferentes capas del tejido gástrico, tal como se evidencia en los estudios histopatológicos del tejido gástrico que hemos realizado en un estudio previo $\left({ }^{13}\right)$.

La producción de GS-NP va a depender de la especie utilizada y la vía de administración. Así tenemos que, a dosis de 1000 y $2000 \mathrm{mg} / \mathrm{kg}$ de Bidens pilosa vía intraduodenal y $0,8 \mathrm{~mL} / \mathrm{kg}$ vía oral de Croton palanostigma, no se observó incremento en la producción de GS-NP $\left({ }^{14}\right)$.

El tratamiento con ranitidina no produjo incremento significativo $(6,6 \%)$ de los grupos GS-NP en la mucosa gástrica, lo cual concuerda con lo encontrado por Álvarez $\left({ }^{14}\right)$ y Sandoval $\left({ }^{9}\right)$, quienes administraron el fármaco vía intraduodenal y orogástrica, respectivamente, a una dosis de 50 $\mathrm{mg} / \mathrm{kg}$ de peso. Sin embargo, en un estudio realizado por Badilla, donde la ranitidina $(50 \mathrm{mg} / \mathrm{kg}$ ) fue administrada vía intraduodenal, se observó un incremento de GS-NP que fue de 10 veces más que el control $\left({ }^{15}\right)$.

Se ha descrito que las prostaglandinas y el óxido nítrico intervienen mayoritariamente en el mantenimiento de la integridad de la mucosa gástrica. El óxido nítrico es gastrocitoprotector, al igual que las prostaglandinas, favoreciendo la estimulación del moco y el mantenimiento del flujo sanguíneo en la mucosa gástrica. Los inhibidores del óxido nítrico exacerban el daño a la mucosa gástrica producido por la indometacina (utilizado también como agente ulcerogénico) y otras drogas antiinflamatorias no esteroideas (AINES), que reducen el flujo sanguíneo a la mucosa y causan la adherencia de los neutrófilos al endotelio vascular $\left({ }^{16}\right)$.

La producción de moco es dosis dependiente, observándose un incremento de $39,4 \%$ y $44,9 \%$ a las dosis de 200 y $400 \mathrm{mg} / \mathrm{kg}$, respectivamente, lo que permitiría una mayor protección. Esta producción incrementada de moco también se ha observado en otras investigaciones, como el de Bidens pilosa a las dosis de 1000 y $2000 \mathrm{mg} / \mathrm{kg}$ (luego de una hora) $\left({ }^{14}\right)$ y con Croton palanostigma vía oral e intraduodenal a la dosis de $0,8 \mathrm{~mL} / \mathrm{kg}$ (después de 4 horas) $\left({ }^{17}\right)$. Sin embargo, en el estudio realizado con extracto de Quassia amara a dosis de 1000 y $1500 \mathrm{mg} / \mathrm{kg}$ vía intraduodenal se observó que la producción de moco no se incrementó en comparación al control (después de 4 horas) $\left({ }^{15}\right)$. Una fracción enriquecida de flavonoide de Alchornea castaneaefolia a la dosis de 100 $\mathrm{mg} / \mathrm{kg}$ no produjo incremento alguno, a pesar que los niveles de prostaglandina hallados a esas dosis fue significativa $\left({ }^{11}\right)$, lo que indicaría que no todas las especies de plantas consideradas antiulcerosas poseen la propiedad de estimular la producción de moco gástrico, ya que esto probablemente dependa de la calidad y cantidad de los metabolitos secundarios presentes en el extracto.

Los niveles de mucus encontrados en el grupo que recibió el tratamiento con ranitidina no fue significativo $(6,8 \%)$, resultados similares a los encontrados por Álvarez y col. $\left({ }^{14}\right)$ y Sandoval y col., quienes utilizaron una dosis de $50 \mathrm{mg} / \mathrm{kg}$, determinando el moco a las dos horas de la administración $\left({ }^{17}\right)$.

Otro de los mecanismos que favorecerían la regeneración de la mucosa gástrica es el aumento 
del pH del jugo gástrico. En nuestro estudio se estimuló la secreción gástrica con la aplicación vía subcutánea de histamina $50 \mu \mathrm{g} / \mathrm{kg}$. Los resultados encontrados demuestran que el tratamiento a las diferentes dosis del extracto hidroalcohólico liofilizado de Bixa orellana no modificó significativamente el $\mathrm{pH}$ del jugo gástrico, con respecto al control, lo que probablemente signifique que los metabolitos secundarios presentes en el extracto no son antagonistas de los receptores $\mathrm{H}_{2}$. Resultados similares, bajo la mismas condiciones, se observó en el estudio con Croton palanostigma $\left({ }^{17}\right)$, en donde la administración del látex vía orogástrica e intraduodenal no modificó el $\mathrm{pH}$ con respecto al control, a diferencia del estudio con diferentes extractos (metanólico $100 \%$, metanólico al $80 \%$ y clorofórmico) de Byrsonia crassa, en el que se observó una disminución del $\mathrm{pH}$ con los dos primeros extractos; y, con el extracto clorofórmico, se mantuvo tan igual como el control $\left({ }^{18}\right)$. Cabe anotar que en este estudio no se utilizó secretagogo.

El pH del jugo gástrico del grupo que recibió el tratamiento con ranitidina se elevó significativamente, lo que comprueba el efecto antagonista de los receptores $\mathrm{H}_{2}$, reduciendo la secreción de $\mathrm{HCl}$ por la mucosa gástrica. Estos resultados también fueron encontrados en el estudio de Badilla y col. $\left({ }^{15}\right)$, Sandoval y col. $\left({ }^{17}\right)$.

En la determinación de la acidez total del jugo gástrico, se puede apreciar que a dosis de 400 $\mathrm{mg} / \mathrm{kg}$ del extracto se incrementó la concentración de $\mathrm{H}^{+}$hasta en $166,6 \%$. En el grupo que recibió el tratamiento con la ranitidina $100 \mathrm{mg} / \mathrm{kg}$, la concentración de $\mathrm{H}^{+}$fue menor a las halladas en los grupos con tratamiento a las diferentes dosis del extracto de Bixa orellana. Esto se debería a que el fármaco compite de forma reversible con la histamina, a nivel de los receptores $\mathrm{H}_{2}$, lo que conlleva a una reducción de la secreción del jugo gástrico y a la disminución de la concentración de hidrogeniones; estos resultados también se encontró en trabajos similares, con los extractos Bidens pilosa $\left({ }^{14}\right)$, Quassia amara $\left({ }^{15}\right)$ y Croton palanostigma $\left({ }^{17}\right)$.

Bajo las condiciones experimentales de nuestro estudio, se puede concluir que el tratamiento con extracto hidroalcohólico de Bixa orellana vía orogástrica tiene un rol citoprotector de la mucosa gástrica, evidenciado por la inducción a la producción de GS-NP y moco en la región glandular del estómago de las ratas a pesar del incremento de la acidez total en la secreción gástrica.

\section{AGRADECIMIENTO}

A los doctores Jorge Arroyo y Salomón Ayala Pío por su apoyo en el desarrollo del trabajo experimental.

\section{REFERENCIAS BIBLIOGRÁFICAS}

1. López-Brea M. Helicobacter pylori: Microbiología, clínica y tratamiento. $1^{\circ}$ Ed. Barcelona: Mosby Doyma Libros; 1995.

2. CYTED/CNP. Metodos de evaluação da atividade farmacológica de plantas medicinais. $2^{\circ}$ ed. Rio de Janeiro: CYTED/CNP; 2001.

3. Placencia M. Evaluación dermatológica de la Buddleia globosa (matico) en el tratamiento de úlcera gástrica inducida en animales de experimentación. Tesis para optar el grado de Magíster. Universidad Nacional Mayor de San Marcos. Lima, Perú. 2001.

4. Placencia M. Actividad antiulcerosa del extracto de Piper angustifolium en animales de experimentación. Lima: CONCYTEC; 1997.

5. Arroyo A. Actividad antiulcerosa de Piper angustifolium (Matico). Tesis para optar el grado de Doctor. Universidad Nacional Mayor de San Marcos, Facultad de Medicina. Lima, Perú. 1998.

6. Ayala S, Díaz D, Palomino M, Armas S, Paz J. Efecto protector de Croton palanostigma y Aloe vera frente a la injuria aguda de mucosa gástrica inducida por etanol en ratas. An Fac Med. 1999;60(1):22-9.

7. Li FE. Actividad biológica del extracto acuoso atomizado de hojas de Bixa orellana L. (achiote) en animales de experimentación. Tesis para optar el grado Académico de Magíster. Universidad Nacional Mayor de San Marcos, Facultad de Medicina. Lima, Perú. 1999.

8. Bernal HY, Correa QJE. Especies vegetales promisorias de los países del convenio Andrés Bello. Bogotá: PREVECAB; 1989.

9. Sandoval M, Ayala S, Oré M, Ricra V, Ugarte G. Incremento del moco gástrico y reducción de grupos sulfhidrilo no proteicos por estimulación con sangre de grado de Croton palanostigma. An Fac Med. 2004;65 Suppl 1:21.

10. Victor BE, Schmidt KL, Smith GS, Reed RL, Thompson DA, Miller TA. Prostaglandin-induced gastric mucosal protection against stress injury. Ann Surg. 1989;209(3):289-96.

11. Hiruma-Lima CA, Calvo T, Rodríguez C, Pezzuto F, Vilegas W, Sousa Brito A. Antiulcerogenic activitiy of 
Alchornea castaneafolea: Effects on somastostatin, gastrin and prostaglandin. J Ethnopharmacology. 2006;104:21524.

12. Juárez S. Caracterização, extração e purificação por cromatografia de compostos de urucum (Bixa orellana L.). Tesis de Maestría. Universidade Federal de Santa Catarina. Florianópolis. 2005.

13. Huamán O. Evaluación farmacológica y bioquímica del efecto protector del extracto hidroalcohólico de hojas de Bixa orellana (achiote) en úlcera gástrica inducida en animales de experimentación. Tesis para optar el grado de Magíster. Universidad Nacional Mayor de San Marcos. Lima, Perú. 2006.

14. Álvarez A, Montero M, Pomar F, Sánchez E. Actividad antiulcerosa de un extracto etanólico de Bidens pilosa L. var. radiata Schult. en ratas. Rev Cubana Plant Med. 1998;3(3):12-7

15. Badilla B, Miranda T, Mora G, Vargas K. Actividad gastrointestinal del extracto acuoso bruto de Quassia amara (simarubaceae). Rev Biol Trop. 1998;46(2):203-10.

16. Angulo P, Díaz D, Espinoza J, Fernández V, Figueroa M, Galarza A. Oxido nítrico, inflamación gastrointestinal y plantas medicinales. Lima: FMV/UNMSM; 2002.
17. Sandoval M, Ayala S, Oré R, Arroyo J. Inducción de la formación de moco gástrico por sangre de grado (Croton palanostigma). An Fac Med. 2002;63(4):251-6.

18. Sannomiya M, Fonseca V, Da Silva M, Rocha L, Dos Santos L, Hiruma-Lima C, et al. Flavonoids and antiulcerogenic activity from Birsonia crassa leaves extracts. J Ethnophatmacology. 2005;97:1-6.

Manuscrito recibido el 17 de octubre de 2007 y aceptado para publicación el 13 de diciembre de 2007.

\section{Correspondencia:}

Mg. Oscar Gustavo Huamán-Gutiérrez

Centro de Investigación de Bioquímica y Nutrición

Facultad de Medicina - UNMSM

Av. Grau 755. Lima 1, Perú

Correo-e: oscarhuaman@hotmail.com 